\title{
Hyperparallel tempering Monte Carlo simulation of polymeric systems
}

\author{
Qiliang Yan and Juan J. de Pablo \\ Department of Chemical Engineering, University of Wisconsin-Madison, Madison, Wisconsin 53706
}

(Received 3 March 2000; accepted 19 April 2000)

\begin{abstract}
A new hyperparallel tempering Monte Carlo method is proposed for simulation of complex fluids, including polymeric systems. The method is based on a combination of the expanded grand canonical ensemble (or simple tempering) and the multidimensional parallel tempering techniques. Its usefulness is established by applying it to polymer solutions and blends with large molecular weights. Our numerical results for long molecules indicate that the new algorithm can be significantly more efficient than previously available techniques. (c) 2000 American Institute of Physics. [S0021-9606(00)50427-9]
\end{abstract}

\section{INTRODUCTION}

Solutions of polymers or biological macromolecules pose significant obstacles to molecular simulation, particularly at low temperatures and elevated densities. Complex fluids such as solutions of macromolecules usually exhibit rugged and complex energy landscapes; it is therefore difficult to sample their configuration space efficiently. Conventional molecular dynamics methods are unable to generate trajectories that are long enough to cover the inherently long characteristic relaxation times that characterize polymeric fluids. Over the last decade, several Monte Carlo methods have been proposed to overcome the barriers facing traditional molecular simulation methods and to improve sampling of configuration space for complex systems. ${ }^{1-11}$ However, most of these methods are of limited use for simulations of polymeric liquids. As an example we mention the simulation of athermal, hard-sphere chains, where most of the above mentioned methods would not apply.

In recent years it has become increasingly clear that open ensembles provide an effective means for overcoming some of the problems associated with slow-relaxation phenomena; in the particular case of polymeric fluids, molecules could get in and out of a system, thereby circumventing diffusional bottlenecks. Furthermore, the expanded grand canonical ensemble Monte Carlo (EGCMC) formalism ${ }^{12,13}$ has rendered open-ensemble simulations of long macromolecules a realistic option.

In a recent paper we drew elements from parallel tempering and configurational bias ${ }^{14,15}$ to propose a new, multidimensional tempering Monte Carlo method for simulation of many-molecule systems. ${ }^{16}$ Such a method combines the benefits of tempering techniques with those of openensemble based simulations. In this paper, we build on that method through a combination of expanded ensembles (also known as simple tempering) and multidimensional parallel tempering. For lack of a better term, we refer to the new method as "hyperparallel tempering" Monte Carlo (HPTMC). The performance of HPTMC is examined in the context of dense polymer solutions and blends. It is shown that HPTMC can be several orders of magnitude more efficient than existing methods for simulation of phase transi- tions. We capitalize on this efficiency to generate phase diagrams for polymer solutions with molecular weights significantly larger than those simulated by previous techniques.

\section{HYPERPARALLEL TEMPERING MONTE CARLO}

Consider a generalized ensemble whose partition function is given by

$$
Z(\mathbf{f})=\sum_{x} \Omega(x) w(x, \mathbf{f}),
$$

where $\mathbf{f}$ denotes a set of specified generalized forces or potentials, which determine the thermodynamic state of the system. In Eq. (1), $x$ is used to denote a microscopic state, or an instant configuration of the system; $\Omega(x)$ is the density of states, and $w(x, \mathbf{f})$ is an arbitrary weighting function for state $x$, at the given set of generalized potentials $\mathbf{f}$. The grand canonical ensemble is recovered by writing

$$
\mathbf{f}=\{T, \mu\}, \quad w(x, \mathbf{f})=\exp (-\beta U(x)+N(x) \beta \mu),
$$

where $\beta=1 / k_{\mathrm{B}} T, T$ is temperature, $\mu$ is the specified chemical potential, $U(x)$ is the potential energy corresponding to configuration $x$, and $N(x)$ is the number of particles in configuration $x$.

We now construct a collective ensemble consisting of $M$, noninteracting replicas of the above mentioned generalized ensemble. Each replica is allowed to be at a different set of generalized potentials. The complete state of the composite ensemble is specified through $\mathbf{x}=\left(x_{1}, x_{2}, \ldots, x_{M}\right)^{T}$, where $x_{i}$ denotes the state of the $i$ th replica. The partition function of the composite ensemble is given by

$$
Z_{c}\left(\mathbf{f}_{1}, \mathbf{f}_{2}, \ldots, \mathbf{f}_{M}\right)=\prod_{i=1}^{M} Z\left(\mathbf{f}_{i}\right),
$$

the unnormalized probability density of the complete state $\mathbf{x}$ is proportional to

$$
p(\mathbf{x})=\prod_{i=1}^{M} \Omega\left(x_{i}\right) w\left(x_{i}, \mathbf{f}_{i}\right) .
$$




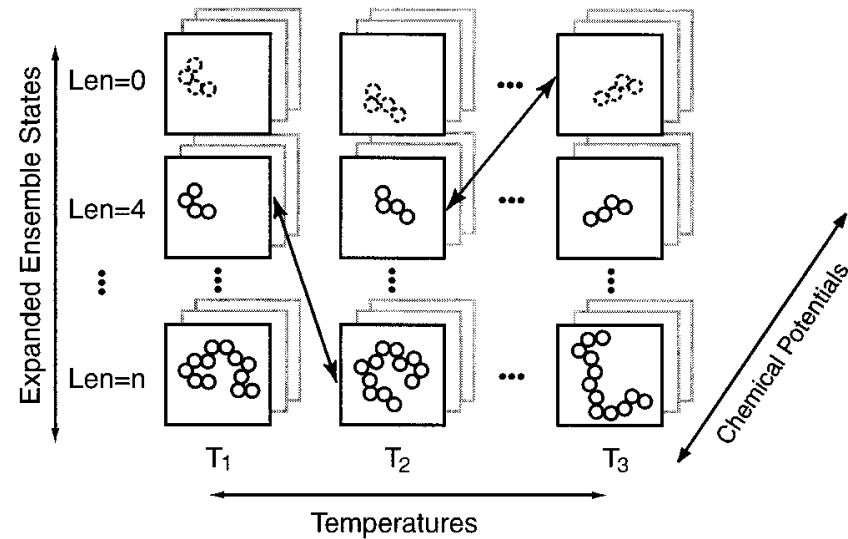

FIG. 1. Schematic illustration of the implementation of hyperparallel Monte Carlo. Each box in the figure represents a distinct replica of the simulated system; these replicas are simulated simultaneously in a single run. In addition to traditional Monte Carlo trial moves, these replicas can (1) change the their state variables in the expanded dimension; (2) exchange configuration with each other, thereby visiting different values of $T$ and $\mu$.

The hyperparallel tempering Monte Carlo method proposed in this work is designed to generate configurations of polymeric systems distributed according to distribution $p(\mathbf{x})$. In this work, we implement the hyperparallel tempering technique in the framework of the expanded grand canonical ensemble, ${ }^{13}$ and we apply it to simulate phase equilibria for polymer solutions and blends. Some of the details concerning the expanded grand canonical ensemble and its application to polymeric systems can be found in the literature. Here we merely mention that the system can jump along a set of expanded states, in addition to the conventional $(N, U)$ phase-space variables of a grand canonical ensemble. For the particular implementation to polymeric fluids, we insert or destroy chain molecules gradually, i.e., several segments at a time. In other words, a simulation box consists of several regular chain molecules and a tagged chain, whose length $n_{y}$ fluctuates during the simulation; $n_{y}$ therefore serves as the expanded state variable. A preweighting factor $\exp (\Psi(y))$ is assigned to each expanded state $y$. In the language of Eq. (1), the weighting function for the expanded grand canonical ensemble is

$\mathbf{f}=\{T, \mu, \Psi\}, \quad w(x, \mathbf{f})=\exp [-\beta U(x)+N(x) \beta \mu+\Psi(y)]$.

If we assume that the segmental chemical potential is independent of chain length, we can set the preweighting function to be

$$
\Psi(y)=\frac{n_{y}}{n} \beta \mu^{r}=\frac{n_{y}}{n}\left[\beta \mu-\ln \left(\frac{N_{y}}{V}\right)\right],
$$

where $N_{y}=N+n_{y} / n ; n_{y}$ is the length of the tagged chain, and $n$ is the length of a full polymer chain. In Eq. (6), $\mu^{r}$ denotes the residual chemical potential of a polymer chain.

Figure 1 illustrates schematically our implementation of HPTMC. Each box in the figure represents a replica in the simulation system; each replica has a different value of $T, \mu$, and $n_{y}$. Three types of trial moves (each corresponding to a distinct axis of the figure) are used to realize the underlying Markov chain.
(1) Canonical Monte Carlo trial moves are used to locally update each of the replicas of the system. Since replicas do not interact with each other, standard Metropolis acceptance/rejection criteria can be employed within each replica.

(2) Trial shrink/growth moves are used to change the length of the tagged chain in each replica, thereby implementing the underlying expanded grand canonical formalism.

(3) Configuration swaps are proposed between pairs of replicas $i$ and $j$, such that

$$
x_{i}^{\text {new }}=x_{j}^{\text {old }}, \quad x_{j}^{\text {new }}=x_{i}^{\text {old }},
$$

To enforce a detailed-balance condition, the pair of replicas to be swapped is selected at random, and the trial swap move is accepted with probability,

$$
p_{\text {acc }}\left(x_{i} \leftrightarrow x_{j}\right)=\min \left[1, \frac{w\left(x_{j}, \mathbf{f}_{i}\right) w\left(x_{i}, \mathbf{f}_{j}\right)}{w\left(x_{i}, \mathbf{f}_{i}\right) w\left(x_{j}, \mathbf{f}_{j}\right)}\right] .
$$

By substituting Eqs. (5) and (6) into Eq. (8), we arrive at the following acceptance criteria for swapping two replicas:

$$
p_{\text {acc }}=\min \left[1, \exp \left(\Delta \beta \Delta U-\Delta N_{y} \Delta(\beta \mu)\right)\right],
$$

where $\Delta \beta=\beta_{i}-\beta_{j}, \quad \Delta U=U_{i}-U_{j}, \quad \Delta N_{y}=N_{y, i}-N_{y, j}$, $\Delta(\beta \mu)=\beta_{i} \mu_{i}-\beta_{j} \mu_{j}$.

\section{MODELS AND SIMULATION DETAILS}

\section{A. Models}

In this paper, we apply the proposed HPTMC method to simulate polymer solutions and blends on a cubic lattice. The polymer is modeled as a chain of connected lattice sites; lattice sites have single occupancy. Nonbonded monomers interact with each other only when they are nearest neighbors. If we denote the interaction energies for polymerpolymer, polymer-solvent, and solvent-solvent pairs by $\epsilon_{p p}, \epsilon_{p s}$, and $\epsilon_{s s}$, respectively, the single relevant energy scale is

$$
\varepsilon=\epsilon_{p p}+\epsilon_{s s}-2 \epsilon_{p s} .
$$

In this work we set $\epsilon_{p p}=-1$, and $\epsilon_{p s}=\epsilon_{s s}=0$.

For pure polymer solutions, we simulate chains consisting of up to 2000 sites. For the lattice model employed in this work, a 2000-site chain corresponds to polystyrene with an approximate molecular weight of one million and a half; though clearly feasible, simulations of longer chains would therefore be of little practical relevance. For polymer blends, we simulated two highly asymmetric systems; in the first, polymers consist of 16 sites and 64 sites, respectively. In the second, polymer chains have 50 sites and 500 sites, respectively. Both blend systems are simulated at constant temperature. The interaction energies are as follows: For system 1, $k T / \epsilon_{11}=2.33, k T / \epsilon_{12}=2.95, k T / \epsilon_{22}=2.80$; for system 2 , $k T / \epsilon_{11}=2.75, k T / \epsilon_{12}=3.10, k T / \epsilon_{22}=3.30$. These parameters are chosen such that the critical temperature for the individual components of the blend are close to each other.

For all systems, each Monte Carlo step consists of 50\% chain growth or shrinking moves, and 50\% local moves (kink-jump and crankshaft moves). Configuration swaps are attempted every 10 Monte Carlo steps. For $n=1000$, the size 
of the simulation box is $L=60$; for $n=2000$, we use a size $L=85$. We use 18 to 20 replicas to calculate the phase diagram of the systems of interest. The temperature and chemical potential of the replicas are such that their states are in the neighborhood of the binodal curve.

\section{B. Optimization of expanded grand canonical ensemble}

The number of segments $n_{c}$ to be grown or deleted during an expanded ensemble trial move is an important parameter in expanded grand-canonical ensemble simulations. We refer to $n_{c}$ as the insertion length. A small value of the insertion length results in an unnecessarily large number of intermediate states, while a large value can lead to low acceptance rates. If $n_{c}=n$, the expanded grand-canonical ensemble reduces to a conventional grand-canonical ensemble. An optimal insertion length can be estimated as follows; for simplicity, we assume that the acceptance rate decreases exponentially as the insertion length increases, that is,

$$
P_{\text {accept }}=a p_{0}^{n_{c}},
$$

where $p_{0}$ is the acceptance ratio when $n_{c}=1$ (i.e., it is the acceptance ratio for a single segment), and where $a$ is a proportionality constant. The length of the tagged chain can increase or decrease with equal probability; the average length of the tagged chain after $N_{s}$ successful growing and shrinking steps should be

$$
\bar{n}=n_{c} \sqrt{N_{s}} .
$$

The average number of attempts to obtain a full-length tagged chain should therefore scale as

$$
\bar{N}=\frac{n^{2}}{a n_{c}^{2} p_{0}^{n_{c}}} .
$$

By minimizing $\bar{N}$, the optimal insertion length is estimated to be

$$
n_{c}^{\mathrm{opt}}=-\frac{2}{\ln p_{0}} .
$$

From the point of view of computational efficiency, the average $\mathrm{CPU}$ time required to insert a full chain is a reasonable measure of performance. If we assume that the CPU time to insert $n_{c}$ segments is proportional to the insertion length, the average $\mathrm{CPU}$ time required to insert a full chain becomes

$$
\bar{t}=\frac{t_{0} n^{2}}{a n_{c} p_{0}^{n_{c}}},
$$

where $t_{0}$ is the $\mathrm{CPU}$ time required to insert one segment. The corresponding optimal insertion length is therefore given by

$$
n_{c}^{\mathrm{opt}^{\prime}}=-\frac{1}{\ln p_{0}} \text {. }
$$

During an actual simulation, chain growth and shrinking moves are not the only elementary steps employed to generate trial configurations; since the CPU time consumed by other types of moves is independent of the insertion length, the actual optimal insertion length is usually between the

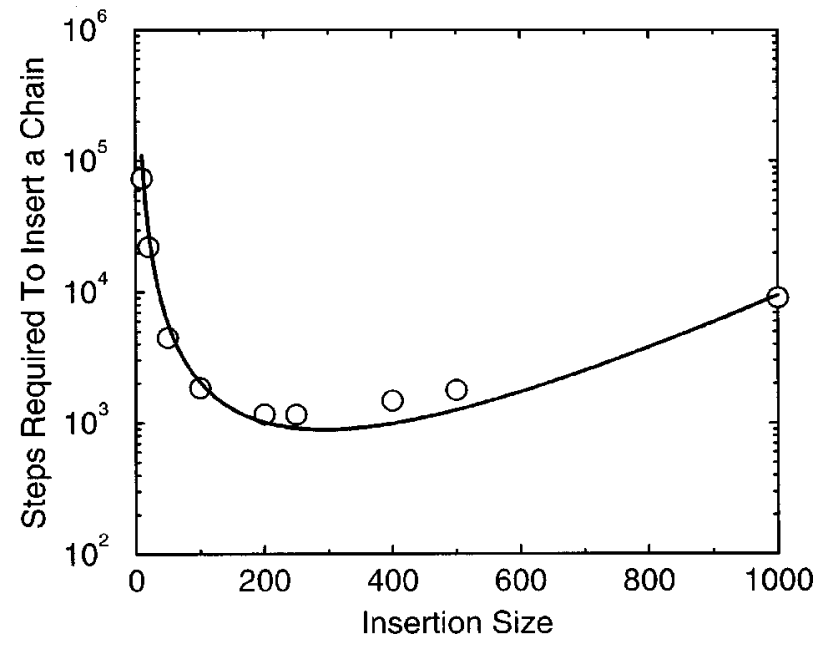

FIG. 2. Average number of trial steps required to generate a full chain or to remove a full chain in expanded grand canonical ensemble simulations, as a function of "insertion size." The symbols are the actual number of steps used in simulations; the curve is a prediction given by Eq. (13).

values given by Eqs. (14) and (16). In our work, a few short preliminary runs with different $n_{c}$ are used to estimate $p_{0}$ from Eq. (11). The value of $n_{c}$ is selected as the integer divider of the chain length which is closest to those given by Eqs. (14) and (16).

To illustrate our optimization procedure, we have calculated the average number of trial growth steps required to insert a whole chain as a function of insertion length for long molecules on a cubic lattice. Figure 2 shows results from a series of simulations for chains of 2000 segments, at $T^{*}$ $=3.34$ and $\beta \mu=300$, where the average density is about $\phi$ $=0.33$. The symbols are the actual average number of steps required to insert a full chain (or delete a full chain) during a simulation; the curve is the prediction of Eq. (13). As we can see, Eq. (13) describes the behavior of our EGCMC simulation reasonably well; the optimal insertion length corresponds to the minimum of that curve, and the location of the theoretical minimum is close to that of the numerical simulation results.

\section{Histogram reweighting}

We use histogram reweighting techniques to determine binodal curves. The details of the technique can be found in the literature. ${ }^{17-19}$ Here we only outline briefly its application to grand-canonical simulations of two-component systems. The probability that, for specified $\left[\mu_{1}, \mu_{2}, V, T\right]$, precisely $N_{1}$ chains of component 1 and $N_{2}$ chains of component 2 are observed having potential energy $U$, is given by

$$
\begin{aligned}
& p\left(N_{1}, N_{2}, U, T, \mu_{1}, \mu_{2}\right) \\
& =\frac{\Omega\left(N_{1}, N_{2}, V, U\right) \exp \left(-\beta U+\beta \mu_{1} N_{1}+\beta \mu_{2} N_{2}\right)}{\Xi\left(\mu_{1}, \mu_{2}, V, T\right)},
\end{aligned}
$$

where $\Omega\left(N_{1}, N_{2}, V, U\right)$ is the microcanonical partition function, and $\Xi$ is the grand partition function, given by 


$$
\begin{aligned}
\Xi\left(\mu_{1}, \mu_{2}, V, T\right)= & \sum_{N_{1}} \sum_{N_{2}} \sum_{U} \Omega\left(N_{1}, N_{2}, V, U\right) \\
& \times \exp \left(-\beta U+N_{1} \beta \mu_{1}+N_{2} \beta \mu_{2}\right) .
\end{aligned}
$$

During a grand canonical simulation at $\left(\mu_{1}^{0}, \mu_{2}^{0}, V, T^{0}\right)$, Monte Carlo estimates of the probability $p$ can be obtained in the form of histograms,

$$
p\left(N_{1}, N_{2}, U, T^{0}, \mu_{1}^{0}, \mu_{2}^{0}\right)=H\left(N_{1}, N_{2}, U\right) / K,
$$

where $H$ is the number of observations (or entries) into a histogram of $\left(N_{1}, N_{2}, U\right)$, and $K$ is the total number of observations in a simulation run.
A Monte Carlo estimate of $\Omega\left(N_{1}, N_{2}, V, U\right)$ is obtained by combining Eqs. (17) and (19) according to

$$
\begin{aligned}
\Omega\left(N_{1}, N_{2}, V, U\right)= & w H\left(N_{1}, N_{2}, U\right) \\
& \times \exp \left(\beta^{0} U-N_{1} \beta^{0} \mu_{1}^{0}-N_{2} \beta^{0} \mu_{2}^{0}\right),
\end{aligned}
$$

where $\quad w=\Xi\left(\mu_{1}^{0}, \mu_{2}^{0}, V, T^{0}\right) / K$ is a constant. Since $\Omega\left(N_{1}, N_{2}, V, U\right)$ is independent of temperature or chemical potential, the probability that the system has $N_{1}$ particles of component $1, N_{2}$ particles of component 2, and energy $U$ at a different state point $\left(T, \mu_{1}, \mu_{2}\right)$, can be estimated according to

$$
p\left(N_{1}, N_{2}, T, \mu_{1}, \mu_{2}\right)=\frac{H\left(N_{1}, N_{2}, U\right) \exp \left[-\left(\beta-\beta^{0}\right) U+N_{1}\left(\beta \mu_{1}-\beta^{0} \mu_{1}^{0}\right)+N_{2}\left(\beta \mu_{2}-\beta^{0} \mu_{2}^{0}\right)\right]}{\sum_{N_{1}, N_{2}, U} H\left(N_{1}, N_{2}, U\right) \exp \left[-\left(\beta-\beta^{0}\right) U+N_{1}\left(\beta \mu_{1}-\beta^{0} \mu_{1}^{0}\right)+N_{2}\left(\beta \mu_{2}-\beta^{0} \mu_{2}^{0}\right)\right]}
$$

The pressure of the system can be determined from

$$
\begin{aligned}
\beta P\left(T, \mu_{1}, \mu_{2}\right)= & \frac{1}{V} \ln \sum_{N_{1}} \sum_{N_{2}} \sum_{U} H\left(N_{1}, N_{2}, U\right) \\
& \times \exp \left[-\left(\beta-\beta^{0}\right) U+N_{1}\left(\beta \mu_{1}-\beta^{0} \mu_{1}^{0}\right)\right. \\
& \left.+N_{2}\left(\beta \mu_{2}-\beta^{0} \mu_{2}^{0}\right)\right]+C,
\end{aligned}
$$

where $C=\ln w / V$ is a constant, which must be evaluated independently. When the system exhibits a phase transition, the above probability distribution can exhibit two or more distinct peaks (if the temperature is well below the critical temperature). To determine phase coexistence (e.g., a binodal curve), the value of the chemical potentials must be manipulated in such a way as to enforce a constant pressure throughout the two (or more) phases present in the system, which is equivalent to the condition that the areas (or volumes) under each peak be equal to each other. The other two conditions for phase coexistence, namely, constant temperature and constant chemical potentials, are satisfied by construction in grand canonical simulations.

The above discussion pertains to a single histogram. Because the ranges of energy and number of particles accessible to a single simulation run are somewhat limited, the ideas discussed above are only useful for small extrapolations in the neighborhood of $\left(T^{0}, \mu_{1}^{0}, \mu_{1}^{0}\right)$. To extend the range of applicability of histogram reweighting techniques, it is therefore customary to run several simulations at different state points, and then combine the resulting multiple histograms to provide a representation of the system over a wide range of conditions. These runs have traditionally been conducted independently of each other. In this work, such simulations are conducted in parallel (i.e., simultaneously) through our proposed HPTMC method. The histograms collected in these simulation runs are subsequently combined to estimate $\Omega\left(N_{1}, N_{2}, V, U\right)$ over a wide range of $\left(N_{1}, N_{2}, U\right)$; such an estimate can in turn be used to calculate thermody- namic properties of interest, including binodal curves. For more details regarding the manipulation of histograms readers are referred to the literature. ${ }^{17-19}$

\section{RESULTS AND DISCUSSION}

Figure 3 illustrates how replicas are swapped during a simulation. The figure can be explained in terms of "physical replicas" and "logical replicas." A "logical replica" is a simulation box which exists at a given thermodynamic state. A physical replica is the actual collection of chains that we follow through the course of a simulation. By construction, a physical replica can occupy any logical replica. Figure 3 shows the evolution of the logical replica at $T^{*}=3.35$ and $\beta \mu=145.9$, for $n=1000$ chain molecules. The ordinate axis indicates which physical replica happens to be visiting the logical replica at any given step during the simulation. A jump in the figure indicates a successful configuration swap. After such a swap, the two relevant logical replicas adopt

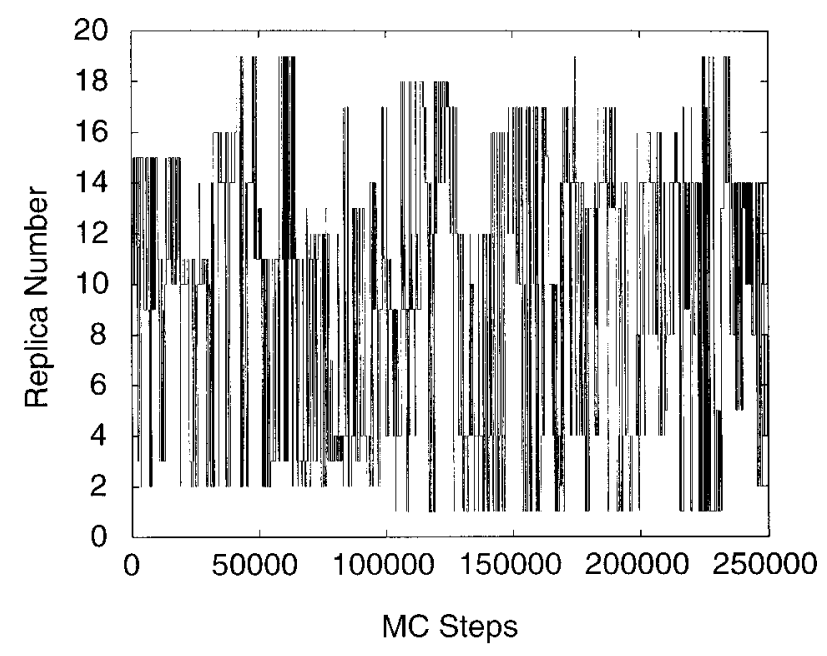

FIG. 3. Replica number as a function of Monte Carlo steps, for $n=1000$, $T^{*}=3.35$, and $\beta \mu=145.9$. 


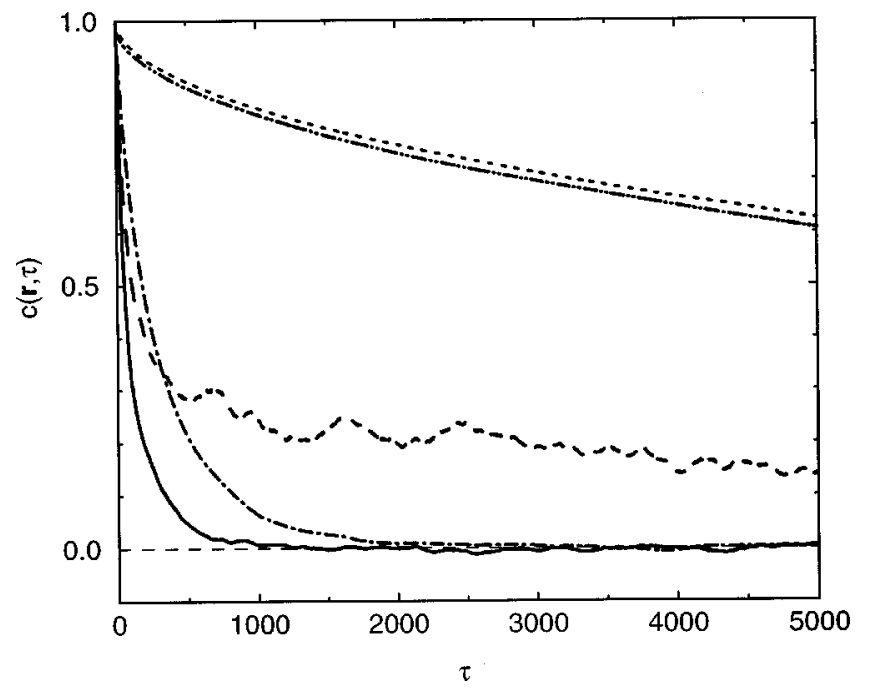

FIG. 4. End-to-end vector autocorrelation function for polymer chains obtained by different simulation methods: (1) short dashed line: canonical ensemble; (2) dashed-dotted-dotted line: grand canonical ensemble; (3) dashed line: multidimensional parallel tempering; (4) dashed-dotted line: expanded grand canonical ensemble; (5) solid line: hyperparallel tempering.

completely new configurations, thereby reducing dramatically the correlation time corresponding to a thermodynamic state. Furthermore, a swap passes a physical replica at a low temperature and a high density over to logical replicas with higher temperature and lower density, where the physical replica can relax more rapidly before eventually returning to its original logical replica. The swapping process greatly accelerates the overall relaxation of the global system and facilitates sampling of phase space under adverse conditions.

In a first test of HPTMC we examine the response of a polymer solution to a step change in chemical potential. In this test, we prepare a set of well-equilibrated initial configurations of $n=1000$ polymers at a relatively low density ( $\phi$ $=0.2$ ), and expose them to a sudden step increase of the chemical potential, for which the new equilibrium density should be about $\phi=0.38$. For reference, a simulation using a traditional grand canonical method is also performed under the same conditions. The results indicate that HPTMC attains the new equilibrium density approximately 50 times faster than the traditional method.

The performance of HPTMC is a result of two, essential elements; expanded-ensemble insertions and destructions, and multi-dimensional replica swapping. To identify the role of these two contributions, we perform a series of simulations using the $N V T$ ensemble, the grand canonical ensemble, multidimensional parallel tempering in a grand canonical ensemble, an expanded grand canonical ensemble, and the newly proposed HPTMC method; in all cases we examine the decay of the end-to-end vector autocorrelation function for the polymer. The decay of this function to zero provides one of the most stringent tests of efficiency for a polymer-simulation technique. Figure 4 shows the results. The dotted curve shows the decay of this function for the $N V T$ ensemble; the relaxation is slow, and reaches a value of only 0.62 after 5000 steps. The curve below that of the $N V T$ ensemble corresponds to the grand canonical simulation; the decay is only marginally better than that for the $N V T$ method. This is due to the extremely low molecule-insertion acceptance rate experienced with macromolecules. The dashed curve shows results from multidimensional parallel tempering simulations in the grand canonical ensemble; the performance is much better than that of naive grand canonical or $N V T$ simulations. A notable feature of this curve is that it decays rapidly during the first 500 steps; after that, the relaxation curve adopts a much smaller slope whose decay to zero is comparable to that for the NVT and GC simulations. Again, the reason for this behavior is that the acceptance rate of the underlying GC simulation is too low. In this case replica swapping does little beyond switching back and forth several nearly-unchanged configurations. The sudden initial drop of the autocorrelation function only arises because several completely different configurations are combined in the Markov chain; swapping, however, is of limited help for the long-time relaxation behavior of long polymeric systems.

The dashed-dotted curve in Fig. 4 shows results for the expanded grand canonical ensemble. As discussed earlier, expanded ensembles improve significantly the performance of a grand canonical simulation. The curve decays to zero after about $3000 \mathrm{MC}$ steps. The performance of the expanded grand-canonical method is more than one order of magnitude better than that of a naive grand canonical simulation. The solid curve shows the results for HPTMC; the decay to zero is even faster than for the expanded grand canonical method. The relaxation of the end-to-end autocorrelation function occurs in less than 1000 steps. In this respect, HPTMC is several times more efficient than the expanded grand canonical technique and it is several orders of magnitude more efficient than the methods that have traditionally been used to simulate polymeric fluids.

It is important to stress the advantages of HPTMC in Fig. 4 over multi-dimensional tempering in the grand canonical ensemble. As discussed in the literature, conventional parallel tempering would not provide a much better performance than a conventional grand-canonical method. Clearly, the high efficiency of HPTMC is largely due to the fact that the underlying expanded-ensemble simulation leads to a high acceptance rate for particle transfers.

Having examined the efficiency of the proposed simulation method, we now proceed to illustrate its usefulness and relevance by applying it to polymeric systems of practical importance. Figure 5 shows coexistence curves for polymers of 100, 600, 1000, and 2000 sites. The lines are the results of this work, and the open symbols are simulation data from the literature. ${ }^{20}$ For $n=100$ and $n=600$, our results are in good agreement with literature reports. Note, however, that with the new method, we are able to explore the phase behavior of long polymer chains down to fairly low temperatures. The computational demands of the new method are relatively modest. For example, calculation of the full phase diagram for polymer chains of length 2000 required less than 5 days on a workstation. The same calculation using traditional grand canonical or Gibbs ensemble techniques would require several years of computer time. ${ }^{21}$ The results of simulations are also consistent with the experimental data for polystyrene-cyclohexane solutions. ${ }^{22}$ More importantly, our 


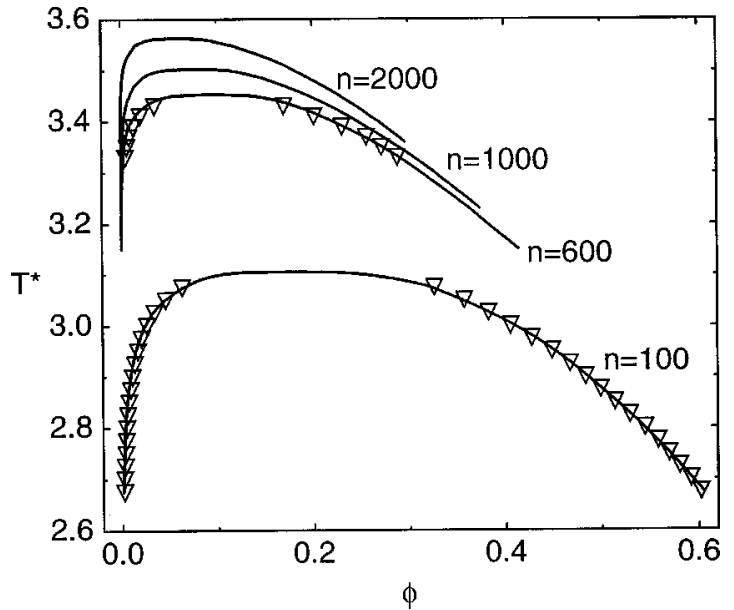

FIG. 5. Phase diagram for long polymer chains. The triangles are results reported by Panagiotopoulos et al. (Ref. 20). The curves show results of this work. Note that with the HPTMC, we are able to calculate the phase diagram for longer polymers, down to lower temperatures, with modest computational requirements.

calculations indicate that, for ultrahigh molecular weights, polymer solutions exhibit a crossover to classical behavior. ${ }^{23}$ This is contrary to the results of previous simulations for shorter polymers, ${ }^{20}$ but it is consistent with recent theoretical arguments by Grassberger and Frauenkron. ${ }^{24}$

In a second example of the usefulness of HPTMC, we use it to examine the miscibility of highly asymmetric polymer blends. For brevity only two systems are considered; in the first, polymers consist of 16 sites and 64 sites, respectively. In the second, polymer chains have 50 sites and 500 sites, respectively. For each replica, the temperature and chemical potential of each component are specified. Threedimensional histograms are collected, and the coexistence curve is determined from a histogram reweighting analysis. Figure 6 shows the projection of the histograms obtained for the $16 / 64$ system on the $N_{1}-N_{2}$ plane. As can be seen in that figure, the simulation conditions for different replicas are such that these histograms overlap nicely with each other, thereby permitting efficient swapping and reliable interpolation via histogram reweighting.

While much theoretical work has been devoted to describe the temperature dependence of miscibility for polymer blends, there are relatively few studies of the effect of pres-

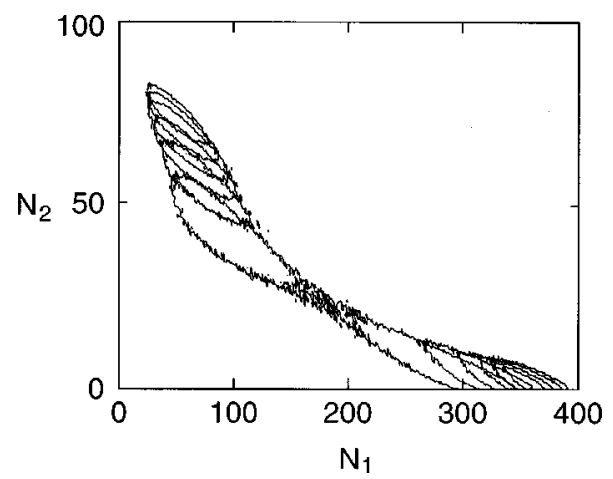

FIG. 6. Histograms obtained in the simulation of polymer blends $(16 / 64$ system). The figure shows the projection of histograms in the $N_{1}-N_{2}$ plane.

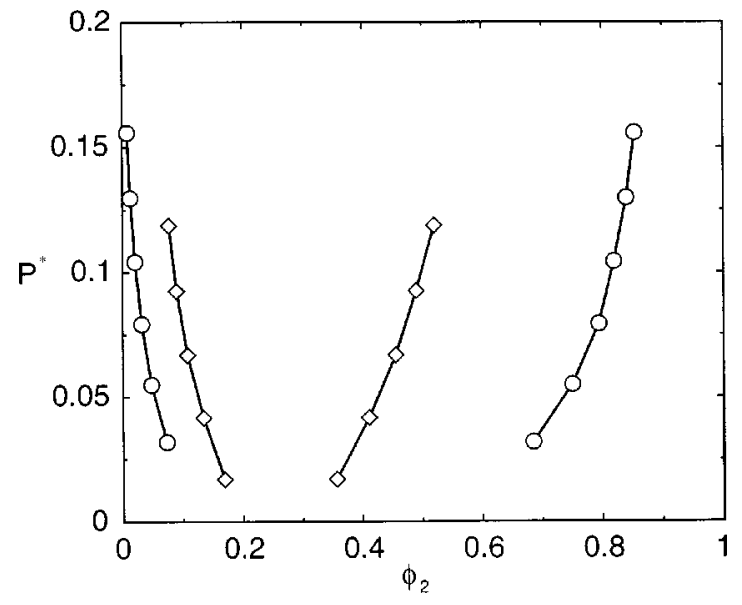

FIG. 7. Phase diagram for asymmetric polymer blends. Circles are for the system with chains of $16 / 64$ sites $\left(k T / \epsilon_{11}=2.33, k T / \epsilon_{22}=2.80, k T / \epsilon_{12}\right.$ $=2.95)$ diamonds are for the system with chains of 50/500 sites $\left(k T / \epsilon_{11}\right.$ $=2.75, k T / \epsilon_{22}=3.30, k T / \epsilon_{12}=3.10$ ).

sure on miscibility. To a large extent, this is due to the fact that most theoretical models assume that polymer blends are incompressible. To address this deficiency, we therefore determine the miscibility of our blends as a function of pressure. Figure 7 shows the resulting phase diagrams. For both systems, pressure has a non-negligible negative effect on the miscibility of the blends; this implies that the volume-change of mixing is positive. Clearly, the assumption of incompressibility for these blends would be invalid. To the best of our knowledge, this is the only available simulation report of the phase diagram of such highly asymmetric and compressible polymer blends. We are therefore unable to provide a comparison between our results and previous work. However, a more extensive simulation study of the miscibility of polymer blends is under way, and in that report we compare our results to the predictions of available theories. ${ }^{25,26}$

The calculations described above have only been possible through use of the HPTMC method. The advantages of this new method have been shown to arise from the combination of biased, open ensemble simulations with replica swapping. The first aspect of the method permits destruction of chain molecules from a simulation box and creation in completely new positions and configurations; one needs not wait for particles to slowly diffuse through the system. Through replica swapping, one takes the whole configuration out of a logical box, and puts a completely different configuration (from another replica) back into the logical box, so that one needs not wait for configurations to slowly diffuse through phase-space bottlenecks. One can think of replica swapping as an arbitrary (but correct) method for forcing fluctuations in phase-space (e.g., fluctuations of energy, density, etc.) into a simulation run. The new method therefore accelerates both real-space and phase-space diffusion, thereby leading to efficient sampling.

Several factors affect the performance of HPTMC. First of all, factors that affect the performance of the underling expanded ensemble simulation clearly influence the performance of HPTMC. In this work, we optimized the performance of the expanded ensemble simulation by carefully se- 
lecting the insertion size. With regards to HPTMC itself, the frequency and success rate of configuration swaps is the most important factor. At first glance, more frequent swaps would appear to increase the efficiency of HPTMC. Too much swapping, however, is detrimental. As Fig. 4 already reveals, if replicas switch too frequently, configurations could be switched back and forth before they relax in their new box. A simple rule-of-thumb that we have adopted is to make the frequency of successful swaps of the same order of magnitude than the frequency of successful particle insertion/removals. Note, however, that simulations of different complex fluids are likely to require some fine tuning to arrive at optimal parallel tempering algorithms for complex fluids.

The acceptance rate for trial swaps depends on the overlap between the distributions of state points for two replicas; the latter depends on how far apart the state points of the replicas are. A higher acceptance rate requires closer state points; in other words, it requires simultaneous simulation of more replicas. Also note that as the systems get larger, the acceptance rate can decrease because the underlying probability distributions become narrower. A possible solution to the problem is to further combine multicanonical sampling into HPTMC; by widening the probability distribution, one should be able to simulate large systems in fewer boxes. Further studies in this direction are currently under way.

In this preliminary work, we have described a novel hyperparallel tempering Monte Carlo method and demonstrated its usefulness with applications to polymer solutions and concentrated polymer blends. For these systems, the proposed method is orders of magnitude more efficient than traditional grand canonical or Gibbs ensemble simulation techniques. More importantly, the new method is remarkably simple and can be incorporated into existing simulation codes with minor modifications. We therefore expect it to find widespread use in simulation of complex, manymolecule systems. One of the few shortcomings of the pro- posed method is the large memory requirement for simultaneous simulation of several boxes. This problem, however, can be turned into an advantage with a parallel processor architecture.

\section{ACKNOWLEDGMENTS}

This work was supported by the Division of Chemical Sciences, Office of Basic Energy Sciences, Office of Sciences, U.S. Department of Energy. Acknowledgement is also made to the Donors of the Petroleum Research Fund, administered by the ACS, for partial support of this research.

${ }^{1}$ B. Berg and T. Neuhaus, Phys. Lett. B 267, 249 (1991).

${ }^{2}$ B. Berg and T. Neuhaus, Phys. Rev. Lett. 68, 9 (1992).

${ }^{3}$ B. Hessebo and R. B. Stinchcombe, Phys. Rev. Lett. 74, 2151 (1995).

${ }^{4}$ A. P. Lyubartsev, A. A. Martinovski, S. V. Shevkunov, and P. N. Vorontsov-Velyaminov, J. Chem. Phys. 86, 1776 (1992).

${ }^{5}$ E. Marinari and G. Parisi, Europhys. Lett. 19, 451 (1992).

${ }^{6}$ D. D. Frantz, D. L. Freeman, and J. D. Doll, J. Chem. Phys. 93, 2769 (1990).

${ }^{7}$ W. Ortiz, A. Perlloni, and G. E. Lopez, Chem. Phys. Lett. 298, 66 (1998).

${ }^{8}$ C. J. Geyer and E. A. Thompson, J. Am. Stat. Assoc. 90, 909 (1995).

${ }^{9}$ M. C. Tesi, E. J. Janse van Rensburg, E. Orlandini, and S. G. Whittington, J. Stat. Phys. 82, 155 (1996).

${ }^{10}$ U. H. E. Hansmann, Chem. Phys. Lett. 281, 140 (1997).

${ }^{11}$ M. G. Wu and M. W. Deem, Mol. Phys. 97, 559 (1999).

${ }^{12}$ F. A. Escobedo and J. J. de Pablo, J. Chem. Phys. 103, 2703 (1995).

${ }^{13}$ F. A. Escobedo and J. J. de Pablo, J. Chem. Phys. 105, 4391 (1996).

${ }^{14}$ J. J. de Pablo, M. Laso, J. I. Siepmann, and U. W. Suter, Mol. Phys. 80, 55 (1993).

${ }^{15}$ D. Frenkel, G. C. A. M. Mooij, and B. Smit, J. Phys.: Condens. Matter 4, 3053 (1992).

${ }^{16}$ Q. Yan and J. J. de Pablo, J. Chem. Phys. 111, 9509 (1999).

${ }_{17}$ A. M. Ferrenberg and R. H. Swendsen, Phys. Rev. Lett. 23, 2635 (1988).

${ }^{18}$ A. M. Ferrenberg and R. H. Swendsen, Phys. Rev. Lett. 63, 1195 (1989).

${ }^{19}$ A. M. Ferrenberg and R. H. Swendsen, Comput. Phys. 3, 101 (1989).

${ }^{20}$ A. Z. Panagiotopoulos and V. Wong, Macromolecules 31, 912 (1998).

${ }^{21}$ A. Z. Panagiotopoulos, Mol. Phys. 61, 813 (1987).

${ }^{22}$ T. Dobashi, M. Nakata, and M. Kaneko, J. Chem. Phys. 72, 6685 (1980).

${ }^{23}$ Q. Yan and J. J. de Pablo, J. Chem. Phys. (submitted).

${ }^{24}$ H. Frauenkron and P. Grassberger, J. Chem. Phys. 107, 9599 (1997).

${ }^{25}$ J. Dudowicz and K. F. Freed, Macromolecules 24, 5074 (1991).

${ }^{26}$ D. G. Gromov and J. J. de Pablo, J. Chem. Phys. 109, 10042 (1998). 